\title{
O crescimento econômico regional de Mato Grosso do Sul
}

\author{
The regional economic growth of Mato Grosso do Sul State in Brazil
}

\section{La croissance économique régionale de l'État du Mato Grosso do Sul au Brésil}

\author{
El crecimiento económico regional de la Provincia de Mato Grosso do Sul en Brasil \\ Jandir Ferrera de Lima* \\ Moacir Piffer* \\ Leandra Aparecida Perego Ostapechen*
}

Recebido em 18/02/2016; revisado e aprovado em 12/06/2016; aceito em 12/09/2016

DOI: http://dx.doi.org/10.20435/1984-042X-2016-v.17-n.4(16)

\begin{abstract}
Resumo: Esse artigo analisa o perfil do crescimento econômico regional do Estado de Mato Grosso do Sul. Para essa análise, foram feitas estimativas quantitativas utilizando dados de Produto Interno Bruto (PIB), população regional, Produto Interno Bruto per capita e a revisão das teorias do crescimento econômico. Os resultados demonstraram que as regiões mais populosas e mais dinâmicas estagnaram, enquanto as regiões periféricas avançaram estimulando a convergência no crescimento econômico regional.
\end{abstract}

Palavras-chave: crescimento econômico; economia sul-mato-grossense; economia regional.

Abstract: This paper analyzes the profile of the economic growth in the regions of Mato Grosso do Sul State in Brazil. For this analysis were made quantitative estimates using gross domestic product data (GDP), regional population and gross domestic product per capita and review of theories of economic growth. The results showed that the most populous and most dynamic regions stagnated, while peripheral regions advanced by stimulating the convergence of the level of regional economic growth.

Key words: economic growth; Mato Grosso do Sul State economics; regional economics.

Résumé: Ce texte analyse le profil du niveau de la croissance économique régionale dans l'État du Mato Grosso do Sul au Brésil. Pour cette analyse ont été faites estimations quantitatives en utilisant les données du produit intérieur brut (PIB), la population régionale et le produit intérieur brut par habitant et l'examen des théories de la croissance économique. Les résultats ont montré que les régions le plus peuplé et ailleurs plus dynamiques ont stagné, mais les régions périphériques ont avancé dans la convergence de la croissance économique régional.

Mots clés: croissance économique; économie de l'Etat du Mato Grosso do Sul; économie régionale.

Resumen: En este artículo se analiza el perfil de crecimiento económico en las regiones de la Provincia de Mato Grosso do Sul en Brasil. Para este análisis se hicieron estimaciones cuantitativas a partir de datos del producto interno bruto (PIB), la población regional y el producto interno bruto per cápita y revisión de las teorías del crecimiento económico. Los resultados mostraron que el crecimiento de las regiones más pobladas y dinámicas se estancó y las regiones periféricas avanzaran estimulando la convergencia regional.

Palabras clave: crecimiento económico; economía de la Provincia de Mato Grosso do Sul; economía regional.

\section{INTRODUÇÃO}

A partir da década de 1980, o Estado de Mato Grosso do Sul começou a se inserir em uma nova fase em sua estrutura econômica. Com o esgotamento da fronteira agrícola, a economia sul-mato-grossense avançou na agrope- cuária de produção mais intensiva, com melhorias na produtividade e ampliação dos excedentes agropecuários exportáveis, além da expansão do turismo ecológico, da produção de celulose e a expansão da produção sucroalcooleira, diversificando e difundindo a sua base econômica.

\footnotetext{
* Universidade Estadual do Oeste do Paraná (UNIOESTE), Toledo, Paraná, Brasil.
} 
Nessa perspectiva, o crescimento econômico regional de Mato Grosso do Sul decorreu da expansão e aumento das atividades de base econômica focadas no setor primário da economia. Ao integrar-se com outras regiões brasileiras e internacionais por meio do comércio inter-regional, as regiões sul-mato-grossenses apresentaram um crescimento da renda real, pela manutenção do dinamismo da (s) atividade (s) de base e da difusão do seu dinamismo para outros ramos produtivos, tanto pela demanda de insumos, como pela demanda complementar de bens e de serviços (PIFFER, 2012).

Frente ao exposto, este artigo analisa o ritmo e o nível de crescimento econômico das microrregiões de Mato Grosso do Sul ${ }^{1}$. Essa análise é descritiva e analítica utilizando o Produto Interno Bruto per capita como variável chave e o primeiro decênio do século XXI como período de estudo. Os resultados e discussões fazem apontamentos sobre as tendências do crescimento econômico no período estudado e servem de elemento para uma discussão da dinâmica da economia sul-mato-grossense.

\section{ELEMENTOS TEÓRICOS E METODOLÓGICOS}

Para Paelinck (1977, p. 160), o crescimento é "um processo de transformações interdependentes que se produzem em certo período". Essas transformações ocorrem na base produtiva e se expressam no crescimento do Produto Interno Bruto ao longo do tempo. Isso caracteriza o crescimento econômico.

Segundo Rostow (2010), o crescimento econômico, por compreender muitas variáveis e tempo de análise, requer um estudo dividido em fases. Essas

\footnotetext{
${ }^{1}$ Este texto apresenta resultados parciais de pesquisa financiada com recursos do Conselho Nacional de Desenvolvimento Científico e Tecnológico (CNPq) e da Fundação Araucária (PR).
}

fases são a preparação para a decolagem, a decolagem propriamente dita e a normalização do crescimento.

A preparação para a decolagem compreende a fase mais longa dos três, podendo levar décadas. Nela se desenvolve um conjunto de atitudes influenciadas por questões socioculturais que culminarão, no futuro, com um sucesso na geração de riqueza, em diversas vertentes distintas. Duas formas clássicas de preparação para a decolagem se conhecem. A primeira delas se dá por transformação política e social de toda ordem, alterando, em alguns casos, também aspectos culturais. A segunda dela não requer as mesmas mudanças da primeira, pois a preparação é facilitada pela abundância de recursos naturais, potenciais geradores de riqueza.

A decolagem, segunda fase, é o momento em que se chega a um forte crescimento econômico, com melhoria de renda, investimentos crescentes e inovações. Pode-se obter essa segunda fase por descobertas tecnológicas e mercados externos favoráveis para negociações lucrativas. Como o lucro se mostra abundante, o reinvestimento e o desenvolvimento em todos os setores da economia simultaneamente caracterizam também essa fase.

A fase da normalização do crescimento, a terceira fase, busca manter os resultados médios da fase anterior e/ ou gradativamente melhorá-los. A manutenção desses cenários favoráveis vai depender da qualidade da tecnologia desenvolvida e utilizada; do equilíbrio social e distribuição da renda; dos investimentos públicos e privados; do comportamento do mercado, das políticas internacionais e das instituições.

Assim, políticas para estimular o crescimento econômico devem estar ligadas ao avanço da tecnologia, às adaptações institucionais e à capacidade de mesclar bases produtivas ou gerar novas bases produtivas ao longo do tempo. Ao longo do tempo, o avanço no cres- 
cimento econômico estimula a transição de uma economia baseada apenas na agricultura para as atividades atreladas à industrialização e ao comércio e serviços (KUZNETS, 1983).

No caso das adaptações institucionais, o crescimento econômico depende da atuação dos agentes econômicos da própria região, aliado aos capitais humano e social. Em alguns casos, estimulados também pela intervenção estatal para ampliar a base produtiva ou melhorar as condições de vida da população (RAIHER,
2012; PIACENTI, 2012). Nesse sentido, o nível e o ritmo de crescimento econômico são indicadores sobre o potencial da estrutura produtiva regional em auferir ganhos e ampliar a acumulação de capital.

\subsection{Procedimentos metodológicos}

O espaço de análise foram as $11 \mathrm{mi}$ crorregiões do o Estado de Mato Grosso do Sul, expostas na Figura 1. A variável base foi o PIB per capita para os anos de 2003, 2007 e 2010.

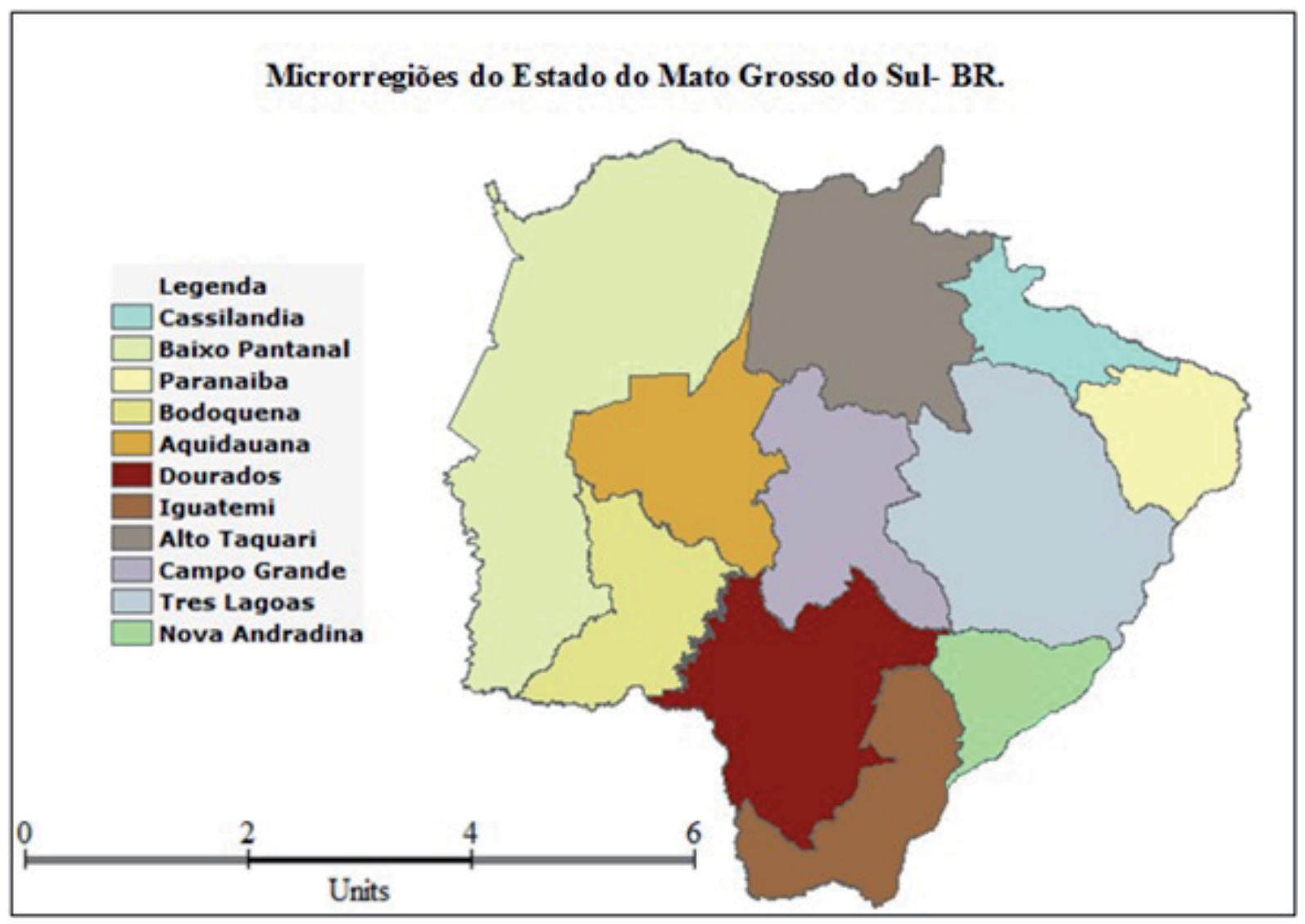

Figura 1 - Microrregiões do Estado de Mato Grosso do Sul - 2015

Fonte: Instituto Brasileiro de Geografia e Estatística (IBGE, 2015).

O uso da variável PIB se explica nas raízes da teoria do crescimento econômico, na qual se afirmava que o desenvolvimento e crescimento econômico eram sinônimos. Quanto maior o PIB per capita, maior a capacidade produtiva da população e, consequentemente, mais apta está a região em termos de acumulação de capital, o que lhe favorece avançar no processo de desenvolvimento econômico em relação às outras regiões. Para a análise dos dados, se utilizou os indicadores de nível e ritmo de crescimento econômico, baseados em Piacenti (2012) e Piacenti e Ferrera de Lima (2012). 
2.1.1 Indicador do Nível de Crescimento Econômico das Microrregiões Sul-matogrossenses (INCMS)

Para a estimativa do nível de crescimento econômico de cada uma das 11 microrregiões do Estado de Mato Grosso do Sul, é construído um indicador baseado no PIB per capita para cada uma delas. O objetivo do indicador é situar cada microrregião em relação ao PIB per capita médio estadual, por meio da equação 1:

$$
\text { INCMS }=\left(\frac{\text { PIBpci }}{\text { PIBpcm }}\right) * 100
$$

Em que:

INCMS = Nível de crescimento econômico dual.

$$
\mathrm{PIB}_{\mathrm{pci}}=\text { PIB per capita da região i; }
$$

$\mathrm{PIB}_{\mathrm{pcm}}=$ PIB per capita médio esta-

A classificação desse indicador se dá da seguinte forma:

Tabela 1 - Classificação do Indicador do Nível de Crescimento Econômico (INC)

\begin{tabular}{cc}
\hline INC & Faixa do INC \\
\hline Significativo & $>100$ \\
Alto & 80 a 99 \\
Médio & 50 a 79 \\
Baixo & 20 a 49 \\
\hline
\end{tabular}

Fonte: Piacenti (2012)

Sendo assim pela classificação do INC, tomando como base o PIB per capita médio de Mato Grosso do Sul, as microrregiões com indicador superior a 100 possuíam naquele ano um PIB per capita superior ao estado. $\mathrm{E}$ as demais, inferior.

\subsubsection{Indicador do Ritmo de}

Crescimento Econômico das Regiões

Sul-mato-grossenses (ICRMS)

Para a determinação do Ritmo de Crescimento econômico das Regiões Sul-mato-grossenses, constrói-se um indicador relativo à média estadual. Demonstrado na equação 2:

$\operatorname{ICRMS}=[((\pi / \psi)-1) /((K / \phi)-1)] * 100$

\section{Em que:} Econômico

ICRMS = Ritmo de Crescimento $\pi=$ PIBPCin $1=$ PIB per capita da região i no período final

$$
\psi=\text { PIBPCin } 0=\text { PIB per capita da }
$$

região i no período inicial

$$
K=\text { PIBPCin1 = PIB per capita }
$$

médio estadual i no período final

$$
\varphi=\text { PIBPCin0 = PIB per capita }
$$

médio estadual i no período inicial

Conforme os trabalhos do Piacenti (2012) e Piacenti e Ferrera de Lima (2012), esse índice possui a seguinte classificação: 
Tabela 2 - Classificação do Indicador de Ritmo de Crescimento (IRC)

\begin{tabular}{cc}
\hline Classificação do IRC & Faixa do IRC \\
\hline Dinâmico & $>100$ \\
Estagnado & 30 a 99 \\
Recessivo & 0 a 29 \\
Depressivo & 0 a -100 \\
\hline
\end{tabular}

Fonte: Piacenti (2012)

Tomando o PIB per capita médio do Estado como 100, as regiões com o IRC superior a 100 obtiveram um ritmo de crescimento superior à média do estado enquanto, nas microrregiões com o indicador inferior a 100, obtiveram um ritmo de crescimento inferior a média do estado, isso significa que o crescimento da população foi superior ao crescimento do PIB. E por fim as microrregiões com o indicador negativo obtiveram uma redução no PIB per capita no período de análise.

\subsubsection{Coeficiente de Williamson}

Para medir as diferenças entre as rendas per capita regionais e em relação à média estadual, utiliza-se o coeficiente de variação de Williamson (1977), demonstrado na equação 3 :

$$
V_{w}=\frac{\sqrt{\sum_{i}\left(y_{i}-Y\right)^{2} * f_{i} / n}}{Y}
$$

Em que:

$V_{w}=$ índice de Williamson

$y_{i}=$ PIB per capita da i-enésima região

$\mathrm{Y}=\mathrm{PIB}$ per capita estadual

$f_{i}=$ população da i-enésima região

$\mathrm{n}=$ população estadual

$\mathrm{V}_{\mathrm{w}}$ é estimado a partir da raiz quadrática do somatório das diferenças entre o PIB per capita de cada região e o PIB per capita do Estado, ponderadas pela população regional sobre a população total, sendo o total dividido pelo PIB per capita Estadual.

Os valores $V_{W}$ variam entre o máximo de um (1) e o mínimo de zero (0), sendo que, quanto mais próximo da unidade, maiores serão as desigualdades regionais de PIB per capita (WILIAMSON, 1977; KON, 2002).

\section{RESULTADOS E DISCUSSÕES}

A figura 2 apresenta a evolução do valor nominal do PIB per capita das microrregiões de Mato Grosso do Sul. Percebe-se que, a partir de 2007, ocorreu a evolução generalizada do PIB per capita na maioria das microrregiões sul-mato-grossenses. No caso, as microrregiões do Baixo Pantanal, Campo Grande e Paranaíba já vinham, desde 2003, com trajetória ascendente de crescimento. Diferente da microrregião de Aquidauana, que, em 2007, reverteu sua trajetória de crescimento e terminou 2010 no mesmo nível de PIB per capita de 2007; as microrregiões de Cassilândia, Iguatemi, Alto Taquari e Dourados, que vinham numa trajetória de queda, reverteram sua posição em 2007 e finalizaram 2010 num ritmo ascendente de crescimento do PIB per capita. Já a microrregião da Bodoquena manteve seu padrão de crescimento do PIB per capita sem grande dinamismo, mas também sem perdas. 


\section{PIB PERCAPITA ESTADUAL E DAS MICROREGIÕES DO MATO GROSSO DO SUL- 2003, 2007 e 2010-em R\$}

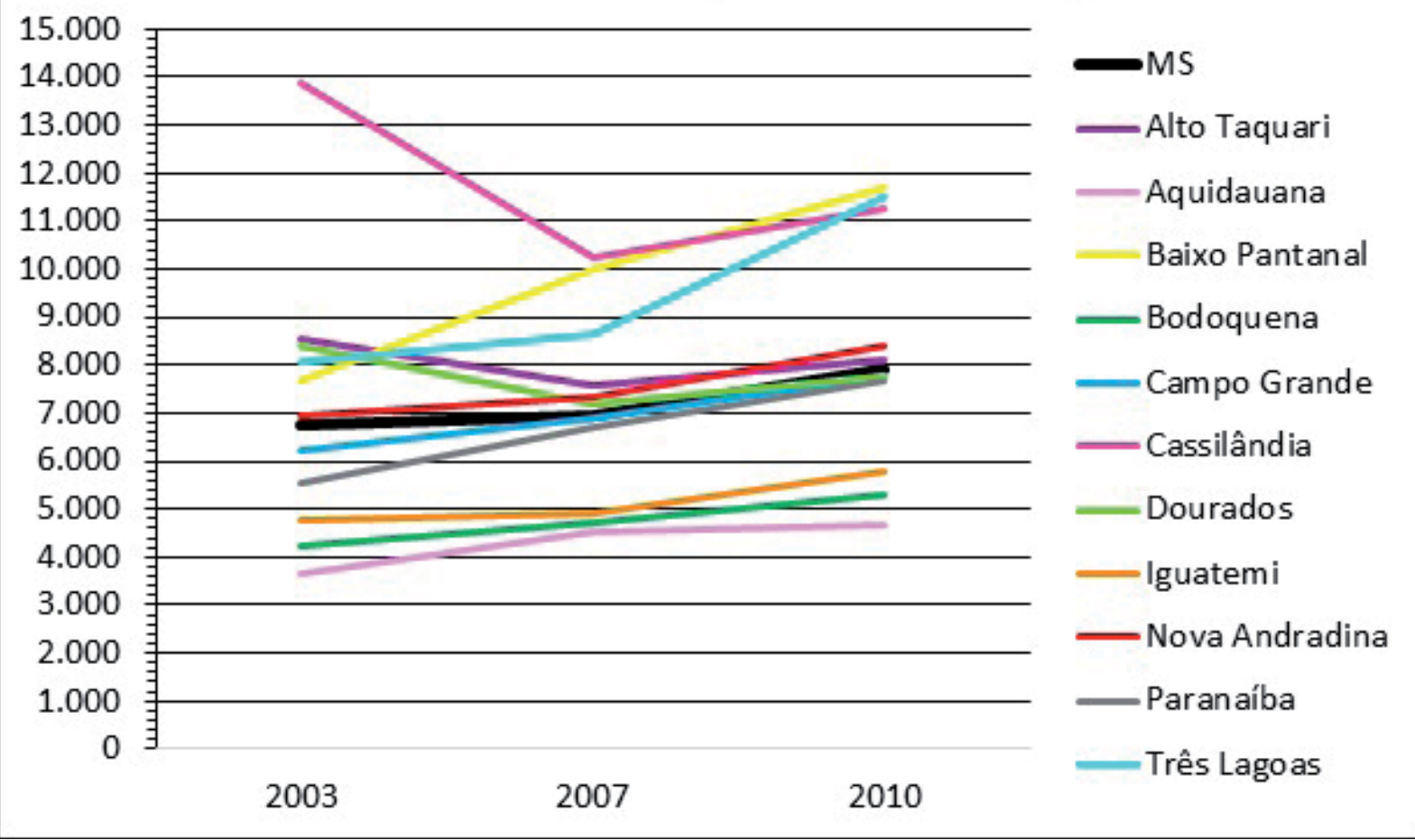

Figura 2 - Evolução do PIB per capita Estadual e das Microrregiões do Estado de Mato Grosso do Sul - 2003, 2007 e 2010 - em R\$

Fonte: IPEADATA (2015).

A tabela 3 apresenta o Nível de Crescimento Econômico das microrregiões de Mato Grosso do Sul. Observa-se que as regiões de Baixo Pantanal, Três
Lagoas, Cassilândia, Nova Andradina e Alto Taquari têm indicador Significativo, ou seja, possuíam um PIB per capita superior ao PIB per capita Estadual.

Tabela 3 - Nível de Crescimento Econômico das microrregiões de Mato Grosso do Sul acima da média Estadual 2003-2007-2010

\begin{tabular}{|l|c|c|c|c|}
\hline \multicolumn{5}{|c|}{ INDICADOR DE NIVEL DE CRESCIMENTO ECONÔMICO } \\
\hline Microrregião de Mato Grosso do Sul & 2003 & 2007 & 2010 & Classificação \\
\hline Baixo Pantanal & 113 & 144 & 148 & Significativo \\
\hline Três Lagoas & 119 & 124 & 145 & Significativo \\
\hline Cassilândia & 205 & 147 & 142 & Significativo \\
\hline Nova Andradina & 103 & 105 & 106 & Significativo \\
\hline Alto Taquari & 126 & 109 & 102 & Significativo \\
\hline Campo Grande & 92 & 99 & 98 & Alto \\
\hline Dourados & 124 & 103 & 98 & Alto \\
\hline Paranaíba & 82 & 96 & 97 & Alto \\
\hline Iguatemi & 70 & 70 & 73 & Médio \\
\hline Bodoquena & 63 & 68 & 67 & Médio \\
\hline Aquidauana & 54 & 65 & 59 & Médio \\
\hline
\end{tabular}

Fonte: resultado da pesquisa a partir de dados do IPEADATA (2015). 
Apesar do indicador de nível de crescimento apresentar uma perspectiva positiva para o contexto das regiões sul-mato-grossenses, percebe-se que as microrregiões de Cassilândia, Alto Taquari e Dourados tiveram uma queda de $-44 \%$, $-26 \%$ e $-23 \%$, respectivamente, em seu Nível de Crescimento, Mesmo assim, se mantiveram em padrão Significativo em relação ao Estado. No caso de Dourados, o reposicionamento do seu nível de crescimento econômico lhe fez se reposicionar no contexto estadual, saindo de um perfil Significativo para Alto. Já Aquidauana perdeu em termos de nível de crescimento na virada do decênio, em torno de $-10 \%$, mas manteve sua classificação.

Tabela 4 - Ritmo de Crescimento Econômico das Microrregiões Sul-mato-grossenses $-2000,2007$ e 2010

\begin{tabular}{|l|c|c|c|}
\hline \multicolumn{4}{|c|}{ INDICADOR DO RITMO DE CRESCIMENTO ECONÔMICO } \\
\hline \multicolumn{1}{|c|}{ Microrregião Mato Grosso do Sul } & $\mathbf{2 0 0 7 - 2 0 0 0}$ & $\mathbf{2 0 1 0 - 2 0 0 7}$ & Classificação \\
\hline Três Lagoas & 133 & 239 & Dinâmico \\
\hline Iguatemi & 122 & 132 & Dinâmico \\
\hline Baixo Pantanal & 323 & 124 & Dinâmico \\
\hline Paranaíba & 111 & 106 & Dinâmico \\
\hline Nova Andradina & 149 & 105 & Dinâmico \\
\hline Campo Grande & 75 & 92 & Estagnado \\
\hline Bodoquena & 100 & 86 & Estagnado \\
\hline Cassilândia & 11 & 71 & Estagnado \\
\hline Dourados & 84 & 60 & Estagnado \\
\hline Alto Taquari & 37 & 51 & Estagnado \\
\hline Aquidauana & 145 & 27 & Recessivo \\
\hline
\end{tabular}

Fonte: resultado da pesquisa a partir de dados do IPEADATA (2015).

As regiões que mais tiveram ganhos significativos em seu nível de crescimento foram Baixo Pantanal com 23\%, Três Lagoas $18 \%$, seguida de Paranaíba, que aumentou $16 \%$ e se mantém no padrão alto de crescimento quando comparado ao do Estado. Por outro lado, em relação a 2003, todas as microrregiões ganharam, mas com magnitudes diferentes.

Ao se comparar com o desempenho do ritmo do crescimento, pode-se ter uma ideia da conjuntura regional e seus desdobramentos frente aos resultados do nível de crescimento econômico. 


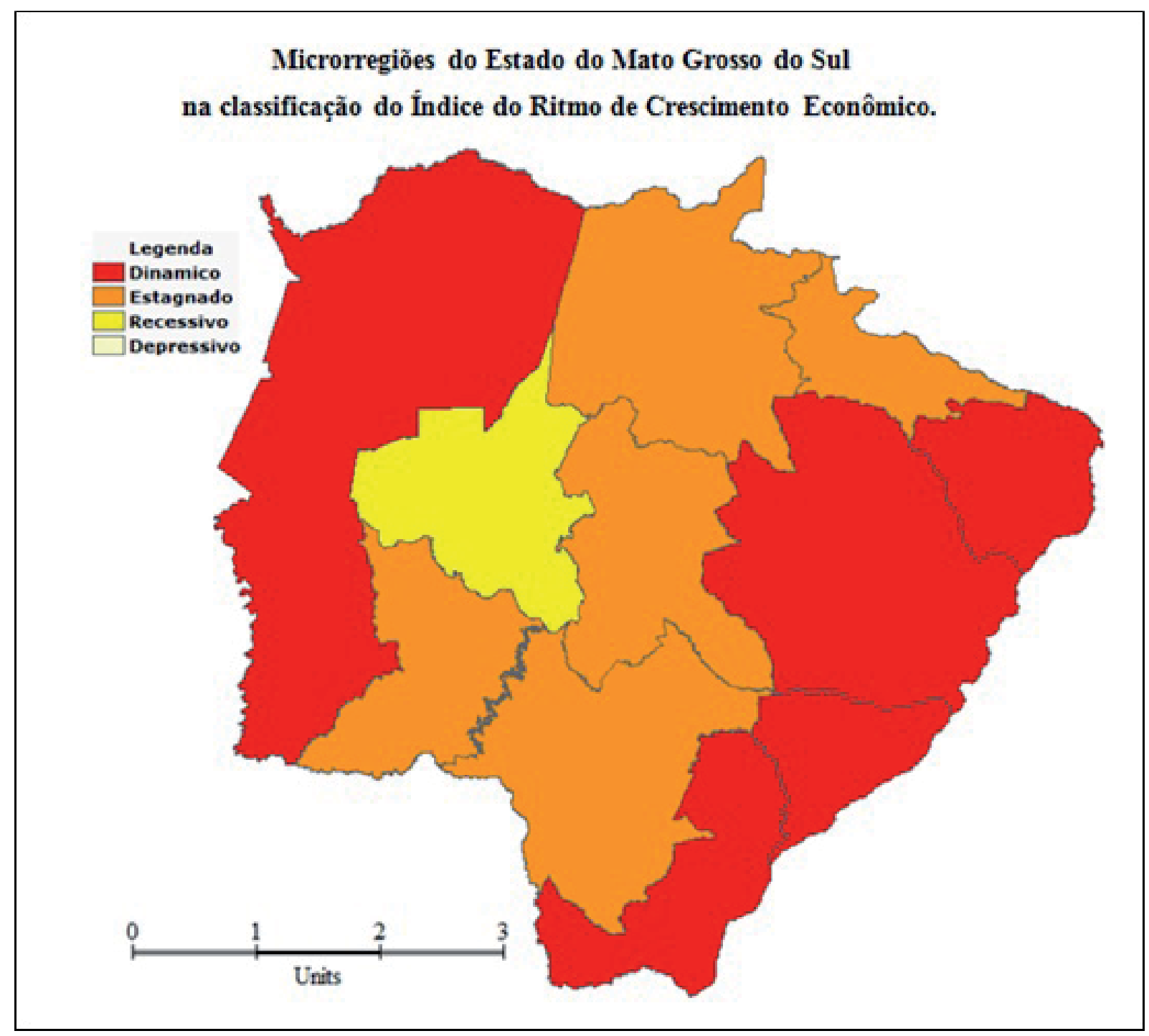

Figura 3 - Ritmo de Crescimento Econômico das Microrregiões do Estado de Mato Grosso do Sul - 2007/2010

Fonte: IBGE (2015).

Ao se comparar os dados das tabelas 3 e 4, percebe-se que, apesar de terem auferido um nível de crescimento razoável em relação à média do Estado, urge pensar em uma política de desenvolvimento regional efetiva no Mato Grosso do Sul. Apesar do nível ter sido de Alto a Significativo, a velocidade em que o nível de crescimento é mantido caiu entre 2010 e 2003. Enquanto as microrregiões que possuem cidades populosas (Dourados e Campo Grande) entram num ritmo de crescimento abaixo da média, a periferia sul-mato-grossense avança. $\mathrm{O}$ dinamismo se transferiu dos núcleos urbanos histo- ricamente mais populosos em direção às áreas periféricas do Estado.

Os resultados do Coeficiente de Williamson demonstraram que a estagnação no ritmo de crescimento das microrregiões de Alto Taquari, Bodoquena, Campo Grande, Cassilândia e Dourados contribuíram para uma maior convergência em termos de nível de crescimento econômico. Enquanto as microrregiões mais populosas e outrora mais dinâmicas estagnavam, outras microrregiões avançavam estimulando a convergência do nível de crescimento econômico. É certo que o ideal em termos de ritmo de 
crescimento econômico não é a estagnação ou o baixo dinamismo, mas garantir, ao longo do tempo, que as microrregiões do Estado fiquem mais homogêneas em termos de dinamismo e desenvolvimento econômico.

Tabela 5 - Indicador de Desigualdade Regional - Coeficiente de Williamson - das Microrregiões de Mato Grosso do Sul - 2003 e 2010

\begin{tabular}{|l|c|c|}
\hline \multicolumn{3}{|c|}{ INDICADOR DE DESIGUALDADE REGIONAL } \\
\hline \multicolumn{1}{|c|}{ Microrregião / MS } & $\mathbf{2 0 0 3}$ & $\mathbf{2 0 1 0}$ \\
\hline Alto Taquari & 0,06 & $0,01 \downarrow$ \\
\hline Paranaíba & 0,03 & $0,01 \downarrow$ \\
\hline Dourados & 0,11 & $0,01 \downarrow$ \\
\hline Nova Andradina & 0,01 & $0,01 \uparrow$ \\
\hline Campo Grande & 0,05 & $0,01 \downarrow$ \\
\hline Cassilândia & 0,16 & $0,07 \downarrow$ \\
\hline Bodoquena & 0,08 & $0,07 \downarrow$ \\
\hline Iguatemi & 0,09 & 0,08 \\
\hline Aquidauana & 0,10 & $0,09 \downarrow$ \\
\hline Baixo Pantanal & 0,03 & $0,11 \uparrow$ \\
\hline Três Lagoas & 0,05 & $0,11 \uparrow$ \\
\hline MS/Centro Oeste & 0,13 & 0,12 \\
\hline MS/Brasil & 0,01 & 0,01 \\
\hline
\end{tabular}

Já os resultados do Coeficiente de Williamson no comparativo entre o Estado de Mato Grosso do Sul e seus pares na região Centro Oeste demonstraram que a distância, em termos de dinamismo regional de Mato Grosso do Sul com relação aos Estados do Centro Oeste brasileiro, não teve variação significativa. Apesar de o Coeficiente ter variado em termos de $8 \%$ em direção a uma maior convergência, percebe-se que, para um período de quase dez anos, ainda foi muito pouco. Cabe lembrar ainda que Mato Grosso do Sul foi o Estado pioneiro a receber as levas de imigrantes no processo de colonização, o que o colocou à frente de outros Estados da Região Centro Oeste em termos de arranque em direção ao progresso. A partir desse resultado, percebe-se que, em relação ao PIB per capita e seu ritmo de crescimento, Mato Grosso do Sul ficou abaixo das expectativas, apesar da sensível melhoria. Já em relação ao Brasil, a situação de Mato Grosso do Sul demonstra estagnação, ou seja, o Estado precisa avançar muito em termos de ritmo e nível de crescimento econômico para se reposicionar positivamente em relação à média brasileira.

\section{CONCLUSÕES}

Este artigo teve como objetivo analisar o ritmo e o nível de Crescimento das microrregiões do Estado de Mato Grosso do Sul entre 2000, 2003, 2007 e 2010, como também a desigualdade regional.

De modo geral, os resultados da pesquisa demonstraram que as microrregiões de Alto Taquari, Bodoquena, Campo Grande, Cassilândia e Dourados estão convergindo para a estagnação no seu ritmo de crescimento. Enquanto as microrregiões mais populosas e outrora mais dinâmicas estagnavam, outras microrregiões avançavam estimulando a convergência do nível de crescimento econômico. O ideal, em termos de ritmo de crescimento econômico, não é a estagnação ou o baixo dinamismo, mas garantir ao longo do tempo que as microrregiões do Estado fiquem mais homogêneas em termos de dinamismo e desenvolvimento econômico. 
Frente ao perfil do ritmo de crescimento econômico das microrregiões do Estado de Mato Grosso do Sul, sugere-se o fortalecimento do potencial de desenvolvimento endógeno dos municípios, em especial por meio de ações e politicas de desenvolvimento territorial. Ações ligadas às potencialidades dos territórios e o empoderamento das comunidades locais estimulam a flexibilização produtiva local, que é considerada uma das formas de se avançar no crescimento e no desenvolvimento econômico.

Diante da análise realizada, constata-se a necessidade de alternativas para solucionar ou, pelo menos, minimizar os problemas detectados em termos de nível e ritmo de crescimento econômico, implementando ações de desenvolvimento endógeno e programas voltados ao fortalecimento das micro e pequenas empresas, que, em geral, são um instrumento importante para o desenvolvimento local, gerador de renda e qualidade de vida à população.

\section{REFERÊNCIAS}

INSTITUTO BRASILEIRO DEGEOGRAFIA E ESTATÍSTICA (IBGE). Base cartográfica. 2015. Disponível em: <http://www.ibge.gov.br>. Acesso em: 20 nov. 2015.

IPEADATA. Base de dados regional. 2015. Disponível em: <http:/ / www.Ipeadata.gov. br>. Acesso em: 28 set. 2015.

KON, Anita. A mensuração da desigualdade do desenvolvimento regional no Brasil. In: KON, Anita (Org.). Unidade e fragmentação: a questão regional no Brasil. São Paulo: Perspectiva, 2002. p. 45-64.

KUZNETS, Simon. Crescimento econômico e desigualdade de rendimento. In: SILVA, Manoela (Org.). Desenvolvimento econômico e repartição de rendimento. Lisboa: Estampa, 1983. p. 21- 50.

PAELINCK, John. A teoria do desenvolvimento regional polarizado. In: SCHWARTZMAN, Jacques (Org.). Economia regional: textos escolhidos. Belo Horizonte: Cedeplar, 1977. p. 157-194.

PIACENTI, Carlos. A. O potencial de desenvolvimento endógeno dos municípios paranaenses. Curitiba, PR: Camões, 2012.

PIACENTI, Carlos; FERRERA DE LIMA, Jandir (Org.). Análise regional: metodologias e indicadores. Curitiba, PR: Camões, 2012.

PIFFER, Moacir. Indicadores de base econômica. In: PIACENTI, Carlos; FERRERA DE LIMA, Jandir (Org.). Análise regional: metodologias e indicadores. Curitiba, PR: Camões, 2012. p. 51-62.

RAIHER, Augusta Pelinski (Org.). Economia paranaense: crescimento e desigualdades regionais. Ponta Grossa, PR: Editora da UEPG, 2012.

ROSTOW, Walt White. A decolagem para o crescimento autossustentado. In: AGARWALA, Angus; SINGH, Paul (Org.). A economia do subdesenvolvimento. 2. ed. Rio de Janeiro: Contraponto, 2010. p. 181-212.

WILLIAMSON, John. Desigualdade regional e o processo de desenvolvimento nacional: descrição e padrões. In: SCHAWTZMAN, Jacques (Org.). Economia regional: textos escolhidos. Belo Horizonte: Editora UFMG, 1977. p. 53-116.

\section{Sobre os autores:}

Jandir Ferrera de Lima: Ph.D. em Desenvolvimento Regional. Professor do PGDRA e PGE da UNIOESTE. Pesquisador e bolsista do CNPq. Pesquisador da Fundação Araucária e da Fundação Parque Tecnológico Itaipu (FPTI-BR). E-mail: jandirbr@yahoo.ca

Moacir Piffer: Professor do PGDRA e PGE da UNIOESTE. Pesquisador do Conselho Nacional de Desenvolvimento Científico e Tecnológico (CNPq). Pesquisador e bolsista da Fundação Araucária. E-mail: mopiffer@yahoo.com.br

Leandra Aparecida Perego Ostapechen: Bolsista de Iniciação Cientifica do Conselho Nacional de Desenvolvimento Científico e Tecnológico (CNPQ). Acadêmica de Ciências Econômicas da Universidade Estadual do Oeste do Paraná (UNIOESTE) - Campus de Toledo. E-mail: leandraperego@hotmail.com 